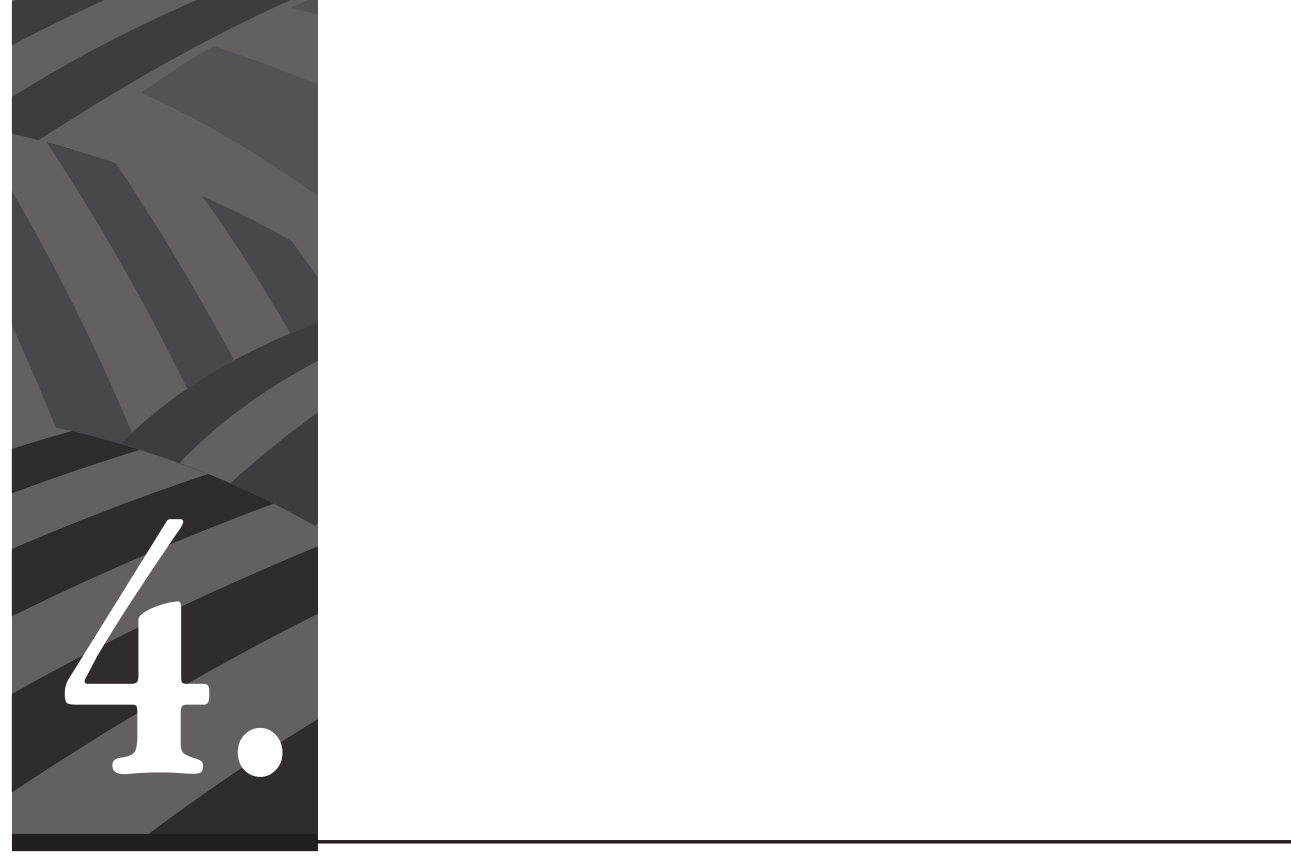

Dinámicas de acaparamiento y concentración de la tierra en Colombia. El caso de la agroindustria de la caña de azúcar 


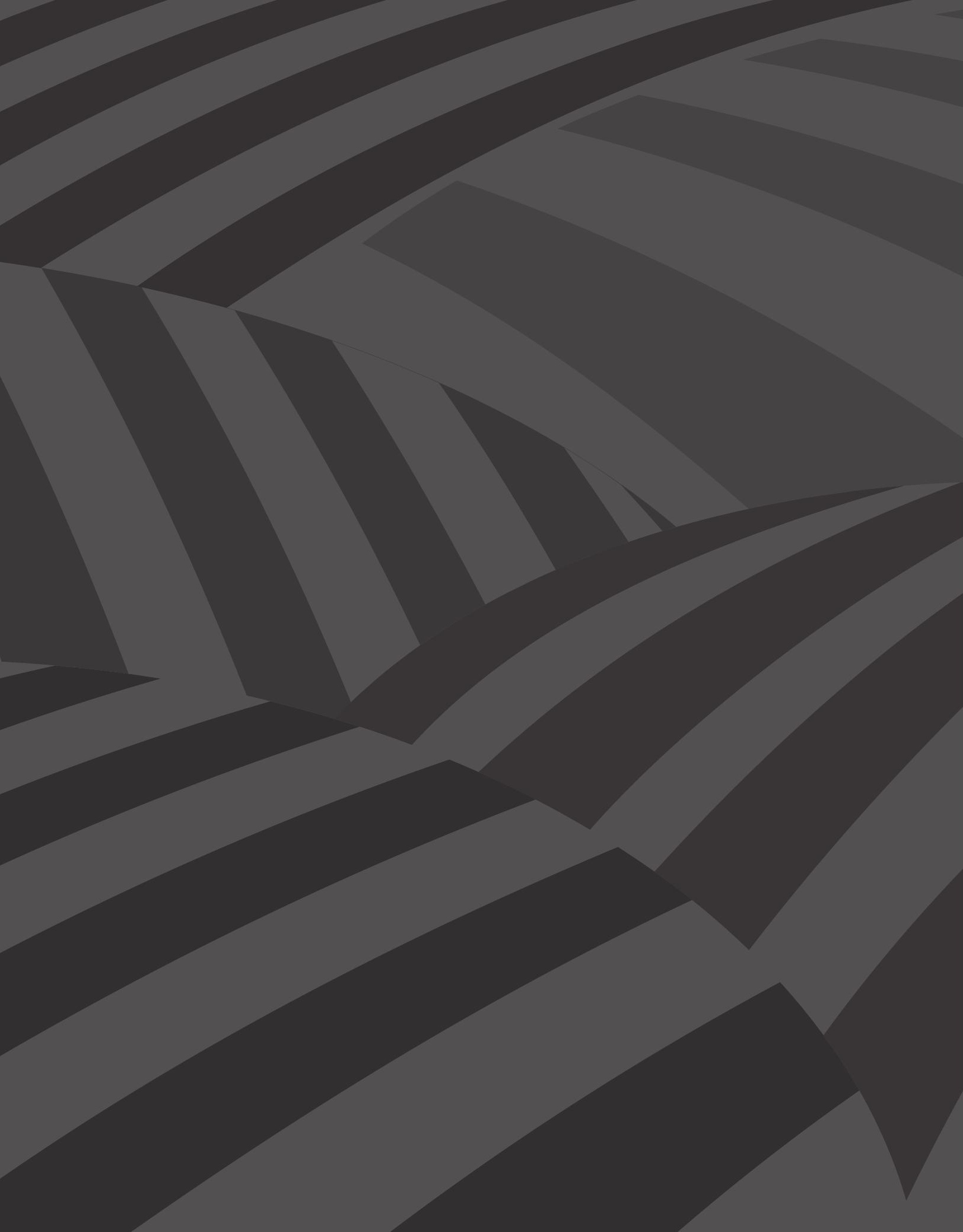




\section{Dinámicas de acaparamiento y concentración de la tierra en Colombia. El caso de la agroindustria de la caña de azúcar}

Por Álvaro Germán Torres Mora*

Resumen: En este texto se aborda el cultivo de caña de azúcar en Colombia y su relación con el acaparamiento y la concentración de la tierra. Con ese objetivo, incorporo el concepto de acumulación por desposesión, que contribuye de manera significativa a comprender la desigualdad de la distribución de la tierra. El análisis del problema involucra la utilización de métodos cuantitativos y espaciales para comparar zonas geográficas que tienen caña de azúcar con aquellos que no la tienen, en aras de determinar el impacto de este cultivo en relación con el acceso a la tierra en Colombian.

Palabras clave: concentración de tierras, acaparamiento de tierras, acumulación por desposesión, biocombustibles, caña de azúcar.

\section{Dynamics of land grabbing and land concentration in Colombia. The case of sugarcane agroindustry}

Abstract: In this text, I discuss the cultivation of sugar cane cultivation in Colombia and its relation to land grabbing and land concentration. Fur such purpose I use the concept of accumulation by dispossession, which contributes significantly to understand the inequality of land distribution in Colombia. The analysis of the problem involves quantitative and spatial methods for comparing geographical areas that have Sugarcane crops with those that do not have them, in order to determine the impact of such crop on land access in Colombia.

Keywords: land concentration, land grabbing, accumulation by dispossession, biofuel, sugar cane.

* $\quad$ Abogado de la Universidad Nacional de Colombia y magíster en estudios sobre el desarrollo de la Universidad de Helsinki, Finlandia. Consultor e investigador independiente en temas de derecho y política agraria. Correo electrónico: germantorresmora@gmail.com. 
Cómo citar este artículo: Torres Mora, Álvaro Germán (2019). Dinámicas de acaparamiento y concentración de la tierra en Colombia. El caso de la agroindustria de la caña de azúcar. Revista Controversia, 212, 107-141.

Fecha de recepción: 8 de diciembre de 2018

Fecha de aprobación: 10 de febrero de 2019

\section{Introducción}

$\mathrm{E}$ ste artículo aborda algunas relaciones entre la agroindustria de la caña de azúcar y el acaparamiento y concentración de la tierra en Colombia. En particular, se analiza el cultivo de dicha especie, que al parecer tiene una relación con la desigualdad en la distribución de la tierra, en términos del coeficiente de Gini. A partir del concepto de acumulación por desposesión se hace una aproximación a esta problemática. El análisis se apoya en datos sobre el índice de Gini a nivel departamental recogidos por la Unidad de Planeación Rural Agropecuaria en el área cultivada de caña de azúcar, según datos del Ministerio de Agricultura.

En Colombia, la concentración histórica de tierras aumentó notablemente entre los años 2000 y 2009. El Gini nacional aumentó de 0.86 a 0.88. Durante este período, las fincas con áreas de más de 500 hectáreas, que hace 20 años ocupaban el $32 \%$ de las tierras útiles, pasaron a ocupar el $62 \%$ de estas y son actualmente propiedad de un $4 \%$ de la población. Según el Estado colombiano, en 2009 el $80.5 \%$ de las parcelas (que pertenecen al 78.3 \% de la población) ocupaba el $10.5 \%$ de la superficie nacional total, mientras que el $52.2 \%$ de la superficie era propiedad del $1.1 \%$ de la población, el equivalente al $0.9 \%$ de las propiedades. El sector agrícola colombiano utiliza menos de 5 millones de hectáreas de las 21.5 millones aptas para la agricultura. El 60 \% son cultivos permanentes y el $33 \%$ son cultivos transitorios. El $68 \%$ son cultivos de rendimiento tardío y el $23 \%$ son plantaciones forestales. En contraste, los ranchos ganaderos ocupan 39 millones de hectáreas, duplicando el área adecuada para tal explotación (20 millones de hectáreas) (Salinas, 2012). 
El Informe Nacional de Desarrollo Humano emitido por el Programa de Naciones Unidas para el Desarrollo (PNUD) (2011) concluye claramente que el modelo de desarrollo rural construido por Colombia es un fracaso, ya que no resuelve los problemas básicos de la sociedad rural. Según el PNUD, el modelo colombiano es injusto, conflictivo y carece de reconocimiento del campesinado. El informe destaca también la sobreexplotación de la ganadería y la consiguiente subutilización de la agricultura; la alta informalidad en los derechos de propiedad, especialmente en el campo; el despojo y abandono de tierras por diversos medios, incluidos los violentos y los contratos viciosos; la persistencia de conflictos sobre la tierra y su relación con el conflicto armado interno; el retraso en la actualización del sistema catastral; la falta de información sobre la tenencia de la tierra y la consolidación de estructuras agrarias ilegales asociadas al conflicto armado (PNUD, 2011).

El prolongado conflicto armado colombiano ha provocado el desplazamiento de millones de personas del campo a las ciudades. Según los registros oficiales, 21698741 personas han sido desplazadas por la fuerza, equivalente al 5 \% de la población total (Ibáñez y Velásquez, 2008). Los departamentos o regiones colombianos con la mayor concentración de tierras tienden a ser los más afectados por el desplazamiento forzado y son el escenario de proyectos importantes como: minería, biocombustibles, agroindustria y agrosilvicultura (Salinas, 2012).

\section{Concentración de tierras y acaparamiento de tierras}

Estos dos conceptos son muy importantes, aunque a veces se usan indistintamente. Por lo tanto, es interesante analizar cómo se han construido.

\section{El acaparamiento de tierras}

La investigación sobre acaparamiento de tierras comenzó después de identificar grandes adquisiciones en África y Asia por parte de gobiernos o empresas extranjeras que buscaban tierras adecuadas para la producción de alimentos. Por lo general, esta situación se apareja con 
un conjunto de instituciones débiles en los lugares donde se vendieron las tierras (Gómez, 2014). El acaparamiento de tierras implica una considerable adquisición de tierras a gran escala, participación de gobiernos extranjeros en estas operaciones y el impacto negativo de tales inversiones en la seguridad alimentaria en los países objetivo (Borras, Franco, Gómez, Kay y Spoor, 2012).

El acaparamiento de tierras es sustancialmente un «acaparamiento de control», lo que significa no solo el control de la tierra, sino también sus recursos inherentes, como el agua. Esta perspectiva, que apunta a superar el enfoque centrado en la propiedad, excluye la necesaria expulsión de campesinos o el despojo de sus tierras. En otras palabras: a pesar de que su tierra sea acaparada, estos pueden permanecer. Al mismo tiempo, superar el enfoque de adquisición implica concentrarse en el análisis del capital invertido, más que en el área adquirida: 500 hectáreas de concesión minera podrían ser tan costosas como 100000 hectáreas de cultivo industrial de árboles (Borras et al., 2012).

El acaparamiento de tierras actual implica inversiones en tierras agrícolas en países ubicados en regiones menos desarrolladas para garantizar la producción de alimentos y combustibles. Además, los gobiernos de estos países a menudo están interesados en atraer inversiones extranjeras para explotar sus tierras (Seo y Rodríguez, 2012). Según Hallam (2009), algunas características importantes del acaparamiento de tierras son:

1. Que el uso de las tierras implica compra o arrendamientos a largo plazo para la producción de alimentos.

2. Una fuerte participación de los Estados de China, Corea del Sur y del Golfo pérsico.

3. Que los principales objetivos son los llamados países en desarrollo.

4. Que los inversionistas privados tienden a ser empresas holding más que expertos en la industria agrícola. 
5. Dado que estas empresas privadas suelen ser financiadas por los gobiernos, no es fácil distinguir la participación de los sectores público y privado en esta materia.

También es importante destacar que el acaparamiento de tierras contemporáneo está inmerso en diferentes crisis, como las de la energía, las finanzas, los alimentos, el combustible, el calentamiento global y otros. Teniendo esto en cuenta, se prefieren aquellos productos que son fácilmente intercambiables y tienen cierto grado de flexibilidad. Por ejemplo, la soya es útil no solo como alimento, sino también como material de alimentación y biodiesel. La caña de azúcar es útil como alimento y para la producción de etanol. El maíz funciona como alimento o para la producción de etanol. El aceite de palma puede usarse como alimento, biodiesel o para aplicaciones industriales (Borras et al., 2012).

El acaparamiento ha sido fuertemente promovido como un mecanismo apropiado para modernizar la producción agrícola e industrial de alimentos y combustibles (White, 2012). Frecuentemente se asume que hay vastas extensiones de tierra disponibles en los países del sur global, por lo que su venta a inversionistas extranjeros es por completo conveniente. De este modo, los capitales extranjeros tienden a privilegiarse por encima de la tierra misma, que de otra manera sería considerada como subutilizada (Matondi, Havnevik y Beyene, 2011). A su vez, hay una tendencia a considerar que estas inversiones son capaces de apalancar empleos, bien sea en el sector agrícola o fuera de este y que las mismas incorporan tecnología foránea suficiente para mejorar la capacidad de producir alimentos (Franco, 2012).

\section{Concentración de la tierra}

La concentración de la tierra puede definirse como un proceso de reconfiguración de la tenencia de la tierra que conduce a una mayor desigualdad en su distribución y acceso. La concentración de la tierra se caracteriza por transferir los derechos de propiedad o tenencia de los pequeños propietarios a un solo titular que explota grandes 
extensiones (Vega, Moncaleano, Ortiz, Carvajalino y Rodríguez, 2017). Otras perspectivas, sin embargo, asumen que el rol de la propiedad en la concentración es variable. En el caso de las inversiones a largo plazo, por ejemplo las forestales, es indispensable la adquisición de la tierra. No sucede lo mismo en materia de cultivos de corto plazo, como la soya, en donde no es necesaria la compra de los terrenos para obtener beneficios económicos (Gómez, 2014).

La distribución de la tierra está asociada al concepto de distribución equitativa, lo que significa que en una población dada cada miembro tiene una fracción proporcional del territorio. Una situación ideal sería aquella donde, por ejemplo, en una población de 500 miembros, cada uno tuviera el 5 \% del área total. Un Gini igual a 1 significa que solo una persona posee el área total; si el Gini es 0 significa una distribución perfectamente equitativa (Rodríguez y Cepeda, 2011).

La región latinoamericana se ha caracterizado por tener una estructura agraria inequitativa, en la que minifundistas o propietarios de pequeñas parcelas y latifundistas están fuertemente extrapolados. Para 1960 los latifundios conformaban el $5 \%$ de las unidades agrícolas, pero alcanzaban un $80 \%$ de la tierra disponible, mientras que los minifundios apenas alcanzaban a sumar el $5 \%$ de la tierra, a pesar de que constituían cuatro quintas partes de esta (Gómez, 2014).

La concentración de la tierra es uno de los problemas más agudos en Colombia. Incluso las cifras oficiales dan cuenta de que la tierra se concentra paulatina y mayormente en zonas dedicadas a la ganadería extensiva, en aquellas con tasas más altas de desplazamiento forzado y aquellas donde el narcotráfico ha generado flujos de capital. Dicha concentración ha sido descrita como un factor clave que impide el establecimiento competitivo de la economía campesina, que a menudo se ve privada de acceso a recursos y tecnología, relegada por completo a espacios geográficos fragmentados (Fajardo, 2001; 2002). 


\section{Diferenciación necesaria de los conceptos}

Como se dijo antes, la literatura contemporánea tiende a alternar los conceptos de acaparamiento y concentración. Sin embargo, debe hacerse un esfuerzo por establecer una diferenciación clara que permita superar, sobre todo, la utilización superficial del concepto de acaparamiento de tierras. Para estos efectos resulta importante utilizar un enfoque histórico, que permite comprender el boom del acaparamiento actual de tierras a partir de la comprensión de un proceso histórico de concentración de tierras necesariamente mediado por relaciones locales de clase. Este enfoque acepta que el acaparamiento de tierras no es un fenómeno reciente, sino que ha ocurrido por ciclos, según las necesidades de acumulación de capital. De acuerdo con este enfoque, el acaparamiento de tierras no se debe únicamente al boom de los commodities, también obedece a un proceso de larga data que involucra a diversos actores sociales en contienda que han producido procesos anteriores de concentración de la tierra (Edelman y León, 2013).

\section{El origen de la concentración de la tierra en Colombia}

Desde los inicios de la República colombiana, el Estado ha promovido los asentamientos a través de la colonización de la tierra, construyendo así una estructura rural dominada por grandes terratenientes. Incluso antes de la independencia de Colombia en 1819, los terratenientes ya habían concentrado vastas cantidades de tierra a través de métodos legales e ilegales de privatización. Sin embargo, grandes extensiones aún permanecían sin ningún título de propiedad, por lo que se convirtieron en objeto de disputas. Algunos de los métodos de apropiación utilizados para adquirir dichas tierras incluyeron, según Machado (2009):

- La venta de baldíos para el pago de la deuda pública.

- La adjudicación de baldíos a miembros de los ejércitos independentistas, ya sea como compensación o como botín de guerra.

- La adjudicación de baldíos a inmigrantes como recompensa por poblar ciertas regiones. 
- Apropiación ilegal de baldíos mediante la ampliación de linderos y la falsificación de títulos originarios.

- La adjudicación de baldíos para exploraciones de quinua, hule y tagua, con fines de exportación.

- La adjudicación de baldíos a empresas extranjeras a cambio de la construcción de infraestructura y urbanización o para la exploraciones petroleras y mineras.

La mayoría de estas tierras fueron otorgadas a empresarios y capitalistas, mientras que los colonos obtuvieron las áreas marginales. Esta práctica, además de las expropiaciones de tierras resultantes de los conflictos armados y las fuerzas del mercado, llevó a una clara concentración. Más importante que el desarrollo socioeconómico para determinar la distribución de la propiedad de la tierra, fue la influencia de grandes terratenientes, comerciantes, políticos y fuerzas militares. Al mismo tiempo, esta situación dio lugar a la perpetuación de los conflictos entre los trabajadores agrícolas sin tierra y su continua incapacidad para salir de la mera agricultura de subsistencia (Machado, 2009).

Los conceptos de acaparamiento y concentración parecen concurrir en muchos aspectos. Sin embargo, para los efectos propios de este texto, el acaparamiento comprende necesariamente la adquisición o el control de tierras con la finalidad específica de adelantar proyectos a gran escala según las necesidades del capitalismo; hoy en día, por ejemplo, existe una fuerte demanda de biocombustibles, minería e infraestructura (Gilbert, 2017). Por otro lado, la concentración de la tierra, como se ha dicho, es un proceso histórico anterior, en el que unos pocos individuos, con base en su capital o influencia, se han apropiado o han controlado amplias extensiones de tierra, a expensas de individuos más pobres.

\section{Acumulación por desposesión}

El concepto de acumulación guarda relación directa con el de acumulación primitiva, ideado inicialmente por Marx (1867). La acumula- 
ción capitalista tuvo que partir de un momento originario en el que la violencia fue decisiva para apropiarse del capital, lo que marcó el final de la era feudal. La nueva burguesía, formada por capitalistas terratenientes, comerciantes y manufactureros, toma el aparato del Estado para su propio beneficio, utilizando leyes para hacerse a las tierras de campesinos, en coalición con grandes productores agrícolas, en un proceso de robo generalizado de propiedad comunal que dejó a miles de campesinos sin sus tierras, destinados a convertirse en vagabundos, mendigos y ladrones. No obstante, es improbable que la acumulación primitiva que ocurrió durante un cierto período de tiempo haya cesado; existe una conexión entre los métodos de acumulación actual y la explotación, como la política colonial, el sistema internacional de préstamos y la guerra. Este proceso se caracteriza por una política de explotación neocolonialista e imperialista, por la reasignación de activos a favor de los capitalistas, por la privatización de las tierras públicas y por el sistema internacional de crédito (Harvey, 2010).

La acumulación primitiva y la acumulación tienen en común el separar a los trabajadores de los medios de producción. Sin embargo, no se trata de entidades conceptuales idénticas. La primera, por definición, es histórica, previa a cualquier acumulación; la segunda, en cambio, supone una reproducción continua del capital (De Angelis, 2000). Aun así, atribuir ese carácter histórico a la acumulación primitiva implica ciertos problemas teóricos en la actualidad. Según Glassman (2006), la acumulación primitiva se observa todavía en espacios rurales contemporáneos en los que la privatización del control de los recursos naturales expulsa a los trabajadores. Por su parte, Ulas (2017) afirma que la acumulación primitiva de ninguna manera es un momento finito, sino que se trata de un proceso cíclico que se reproduce en escenarios de capitalismo actual.

Hoy en día, la acumulación primitiva ocurre en áreas periféricas fuera del núcleo del capitalismo. Esto se puede ver en la China contemporánea, donde el antiguo pueblo agrario se ha convertido en una población urbana de bajos salarios. También en algunas zonas de África, América 
Latina y el sur y este de Asia, donde la extracción de recursos naturales conduce a una expropiación violenta de los campesinos. Lo mismo se puede observar en la India, donde la creación de “zonas económicas especiales” ha fomentado la expulsión de las poblaciones campesinas para permitir el asentamiento de agroindustrias en condiciones privilegiadas (Harvey, 2010). Sin embargo, estas tácticas modernas, que pueden incluir los procesos de privatización, se entienden mejor utilizando el concepto de acumulación por desposesión.

Según Harvey (2003), poner la acumulación en una etapa original o primitiva de la historia podría llevar a disminuir su relevancia actual, dada la persistencia de las prácticas depredadoras dentro del capitalismo: el desplazamiento de campesinos locales, la formación de poblaciones sin tierra y la privatización de bienes sociales son solo algunos ejemplos. Por lo tanto, un enfoque moderno debe sustituir los términos tradicionales. Resulta más pertinente usar el concepto acumulación por desposesión.

Este proceso de acumulación contemporáneo involucra necesariamente una coerción extraeconómica, que, en el caso de la tierra, supone la participación activa de un agente coercitivo, a saber, terratenientes, mafias, paramilitares y, más frecuentemente, los Estados (Levien 2011a; 2012; 2013). La acumulación por desposesión puede servir para la comprensión del fenómeno de acaparamiento de tierras, comoquiera que la expropiación se efectúa por medio de mecanismos distintos a los regulados en el mercado. Las tierras son expropiadas por los Estados sin la voluntad de los propietarios (Levien, 2012). Estos son utilizados por los capitalistas debido a que son los que cuentan con el poder para adelantar expropiaciones cuando los campesinos no desean vender sus inmuebles (Harvey, 2006).

\section{Dinámicas del acaparamiento de la tierra en Colombia}

Diferentes factores juegan un papel en la concentración de la tierra en Colombia, como el narcotráfico, el despojo violento y el uso de las tierras 
como reserva de valor o instrumento de poder (Ibáñez, 2012). Esto es parcialmente confirmado por la Organización para la Alimentación y la Agricultura (FAO, por sus siglas en inglés) en un estudio realizado en América Latina a partir del 2010: a diferencia de otros escenarios de acaparamiento de tierras, el caso colombiano se caracteriza por la violencia, los conflictos rurales, el desplazamiento forzado y la apropiación de tierras por parte de grandes terratenientes, empresarios, paramilitares y traficantes de drogas. Este fenómeno se ha visto agravado por los incentivos estatales a las inversiones privadas en recursos mineros y energéticos, agrocombustibles, maíz y silvicultura (Salinas, 2012).

\section{Principales aspectos de la concentración de la tierra en Colombia}

La concentración histórica colombiana posterior a la adjudicación de baldíos se ha relacionado tradicionalmente con la ganadería. La utilización para tales propósitos claramente excede la capacidad de la tierra en Colombia. Solo 20 millones de hectáreas son aptas para criar ganado, pero dicha actividad ocupaba 39 millones de hectáreas en 2009. Sin embargo, en línea con las tendencias mundiales, la concentración de la tierra en Colombia parece asociarse progresivamente a la minería y a las agroindustrias, cuya inversión es altamente apoyada por el Estado colombiano. De hecho, en 2009 se adjudicaron 8.5 millones de hectáreas como concesiones mineras y se solicitaron 30 millones más para los mismos fines (Salinas, 2012).

\section{Negocio de la caña de azúcar en Colombia}

La caña de azúcar se ha cultivado principalmente en Cauca, Risaralda y el Valle del Cauca. En el año 2002 ocupaba 200000 hectáreas. Su producción se distribuye en 13 ingenios azucareros diferentes, 40 empresas que procesan alimentos y bebidas, 2 generadores de energía eléctrica, 1 empresa procesadora de papel y 3 empresas de extracción de sustancias químicas (CEPAL, 2002).

El proceso ha sido particularmente importante en el Valle del Cauca, departamento que en el siglo XX pasó de enfocarse en las actividades 
agrícolas y ganaderas al monocultivo de la caña. Entre 1851 y 1920 las comunidades negras consolidaron una economía basada en minifundios de no más de 3 hectáreas, dedicados principalmente a la producción de cacao (Hurtado, 2001). Sin embargo, los cultivos a gran escala de caña de azúcar terminarían imponiéndose, debido en gran parte a la inversión de grandes capitales agroindustriales, dinámicas de urbanización y a la fuerte participación de élites locales que impulsaron a la agroindustria como motor del desarrollo (Uribe-Castro, 2014). Esta transformación requirió que las comunidades campesinas del norte del Cauca vendieran sus tierras a los ingenios azucareros y se convirtieran en mano de obra, dentro de lo cual también ejercieron como corteros, sobre todo a partir de 1950 y hasta 1985, lo que, a su vez, crearía las condiciones suficientes para industrializar la región a partir de 1997. Valga anotar que las élites terratenientes tuvieron una fuerte participación mediante el aprovechamiento de los hechos de inestabilidad propios de la época de la violencia para así acaparar tierras de las comunidades negras. Para lograrlo promovieron los proyectos agroindustriales entre las poblaciones afrodescendientes, haciéndoles creer que los cultivos transitorios generaban más ingresos, sin obviar que también inundaron y quemaron grandes extensiones de tierras (Escuela Itinerante Afronortecaucana y Grupo Semillas, 2013).

Ayala-Osorio (2019) destaca que la caña de azúcar ha sido capaz de transformar el territorio del Valle del Cauca mediante la supresión de las anteriores territorialidades y su remplazo por modelos industriales. En este proceso tuvo mucho que ver un modelo eurocéntrico que menosprecia las culturas tropicales. Las élites blancas perciben a los afrocolombianos como simples campesinos pobres anclados a lógicas precapitalistas que deben ser modernizadas.

Las explotaciones de la caña de azúcar podrían implicar algunos valores agregados dados sus múltiples usos, según la CEPAL (2002):

- La industria sucroquímica la utiliza como materia prima para producir licores, alcohol, ácido cítrico, levaduras, gas carbónico, acetatos, carbonato de calcio y fertilizantes. 
- La industria de confitería y chocolate, formada por al menos 50 empresas en Colombia, utiliza suministros compuestos hasta en un 80 \% por azúcar.

- La industria del papel utiliza la fibra de caña de azúcar como su principal materia prima. La compañía colombiana Propal es sobresaliente, pues es una de las 5 más grandes del mundo que utiliza fibra de caña de azúcar. Gracias al papel y al cartón, aproximadamente 40 empresas trabajan y forman el grupo de artes gráficas, liderado por el grupo Carvajal, que tiene plantas de producción propias y actividades en más de 18 países.

- Sirve para la producción de energía, para la cual se utiliza la mayor parte de la fibra de caña (80 \%). Esta genera más de 80 MW por año.

- Los jarabes se utilizan como fertilizantes y como alimento para animales.

La industria colombiana de la caña de azúcar está dominada por estos grupos industriales, según datos de la CEPAL (2002):

1. Manuelita: fundada en 1863 y pionera de la explotación azucarera en Colombia. Este grupo también cultiva palma aceitera en la región de la Orinoquía y realiza otras actividades como el cultivo de camarón para exportación. Para 2002, Manuelita era propietaria de 22000 hectáreas utilizadas para cultivos de caña de azúcar.

2. Grupo Ardila Lulle: propietario de un canal de televisión privado (RCN), una cadena de estaciones de radio, una empresa de envases de vidrio (Peldar) y Sucroal, que produce alcohol etílico, ácido cítrico, levadura, yeso, ácido acético, vinagre, dióxido de carbono, acetatos, carbonato de calcio y abonos agrícolas y otros. El grupo Ardila Lulle es propietario de un negocio de refrescos (Postobón) que compite con Coca Cola en Colombia; de ahí su interés en la producción de azúcar. Debido a los altos costos del azúcar, el grupo decidió tomar el control de los ingenios azucareros Cauca, Providencia y Risaralda desde los años setenta. El grupo es el mayor productor de azúcar del país, con 20000 hectáreas de caña de azúcar en el 2002, cuya transformación se hace a través de Incauca.

3. Grupo Riopaila: fundado en 1931 en el departamento del Valle para la producción de confitería, es el propietario de Colombina, una empresa 
colombiana líder en el sector de confitería. Este grupo proporciona azúcar tanto para el mercado interno como para el internacional y utiliza su producción para sus propias empresas, que se centran en la producción de dulces, chocolates, galletas y mermeladas. Sus plantaciones de caña de azúcar cubren 20000 hectáreas.

Para el 2002, el cúmulo colombiano de caña de azúcar ocupaba 207 462 hectáreas. El 77 \% de estos están ubicados en el departamento de Valle y el $20 \%$ en el departamento de Cauca, lo que resulta en cluster alrededor del río Cauca. Además de este grupo, el área cultivada total en el país es de 429000 hectáreas (DANE, 2015). Según el Ministerio de Agricultura y Desarrollo Rural (MADR) (2016), fueron 255612 en 2016.

\section{Transformación de la caña de azúcar}

La caña de azúcar es la única fuente de producción de alcohol carburante en Colombia a pesar de que puede ser obtenido a partir de varias materias primas. Sin embargo, la caña de azúcar ha demostrado ser la más productiva y la que tiene el mejor rendimiento. Pocos materiales pueden competir con la caña de azúcar debido a sus condiciones económicas de acuerdo con la geografía colombiana y la infraestructura y logística ya instaladas, especialmente en el departamento del Valle. También es importante destacar la presencia de tales plantas en Puerto López, en la altillanura colombiana (Delgado, Salgado y Pérez, 2015).

\section{Fomento a los biocombustibles en Colombia}

El Estado colombiano ha incentivado en gran medida la producción de agrocombustibles mediante la emisión de normas relevantes, algunas de las cuales se describen aquí:

1. La Ley 693 del 2001 ordena el uso de biocombustibles mezclados con la gasolina que se consume en centros urbanos de más de 500000 habitantes, de acuerdo con los requisitos del Ministerio de Minería y Energía.

2. La Ley 939 del 2004 promueve la producción de biocombustibles a partir de fuentes vegetales o animales, permitiendo su uso en motores diésel y eximiéndolos del IVA. 
3. El Decreto 383 del 2007 apoya los proyectos agroindustriales reduciendo en un 50 \% la inversión requerida para el reconocimiento de zonas de libre comercio. Los proyectos regulares deben invertir al menos 150000 salarios mensuales mínimos, mientras que los agroindustriales deben invertir solo 75000 .

4. El Decreto 2629 del 2007 ordena que los vehículos nuevos deben adaptarse para usar combustibles hechos con 80 \% de gasolinas básicas y $20 \%$ de alcoholes carburantes. Esta regulación fue modificada por el Decreto 1135 del 2009, que promueve el uso de los sistemas de combustible flexible (E85), es decir, aquellos que permiten que los vehículos funcionen utilizando mezclas hechas de aceites fósiles y al menos $85 \%$ de alcohol carburante. Mediante este decreto, el gobierno ordenó que para el $1^{\circ}$ de enero del 2016, aquellas marcas que producen y comercializan en el mercado colombiano deben adaptar el $100 \%$ de sus vehículos con motores de hasta $2000 \mathrm{~cm}^{3}$ para que usen E85.

5. El Decreto 2594 del 2007 crea el Fondo para inversiones de riesgo, cuyos objetivos son apoyar y desarrollar iniciativas productivas en zonas con bajas inversiones privadas, priorizando proyectos agroindustriales. El fondo, formado por recursos del presupuesto nacional, invierte en proyectos específicos, ya sea mediante la capitalización de las empresas que realizan dichas actividades o mediante la participación directa en ellos.

6. El Decreto 4892 del 2011 ordena que toda la gasolina consumida en el país debe ser una mezcla compuesta de un $10 \%$ de alcohol y el resto de gasolina básica.

La demanda de etanol combustible está parcialmente cubierta en Colombia; hasta el $82 \%$ del etanol consumido se produce internamente (260-300 millones de litros). Sin embargo, no es suficiente para cumplir con el requisito nacional E10 (90 \% de gasolina; $10 \%$ de etanol). Esta situación ha alentado a las empresas a hacer lobby para aumentar la producción. En cualquier caso, regulaciones como el Decreto 1135 del 2009 que ordena el uso de E85 en vehículos de hasta $2000 \mathrm{~cm}^{3}$ obligan a la producción de biocombustibles (Delgado et al., 2015).

\section{Medición de la concentración de tierras en Colombia}

La medición de la concentración de tierras en Colombia requiere del uso del catastro nacional, que incluya las tierras que son de propiedad 
privada, utilizado principalmente para fines de tributación (IGAC, 2011). Para cumplir su misión, el catastro nacional incluye datos de las oficinas registro e instrumentos públicos, que registran las transacciones de tierras (Ley 1579 del 2012). Sin embargo, esta información podría estar sesgada por la alta informalidad de la tierra en Colombia, donde el 52.2 \% de las tierras carecen de títulos de propiedad (Perfetti y Saavedra, 2017). Dicha informalidad se ha asociado con altos costos de registro, que a menudo es imposible para los campesinos (Vogelgesang, 2003). Teniendo en cuenta este problema, una medida razonable basada en el catastro nacional encontró que, para el año 2000, el $97.1 \%$ de los propietarios poseen el $41.6 \%$ del área total; en otras palabras, el 58.4 $\%$ de la tierra es propiedad de $2.9 \%$ de los propietarios. Ampliando los rangos, el $80 \%$ de la tierra es propiedad de $10 \%$ de los propietarios. En el mismo año, el índice nacional Gini era de 0.8479 , lo que muestra una alta concentración de tierra (Rodríguez y Cepeda, 2011).

Según Ibáñez et al. (2012), algunos cálculos incluyen a Rincón, quien afirma que el índice de Gini subió de 0.85 en 1984 a 0.88 en 1996; Heath y Deininger, quienes concluyeron que disminuyó de 0.86 en 1960 a 0.84 en 1988; y Deininger y Lavadenz, quienes concluyeron que para 2003 el índice era de 0.85 .

\section{Análisis de datos}

Usando datos de la Unidad de Planificación Rural Agropecuaria (UPRA) (2016) sobre la concentración de la tierra en Colombia y del MADR sobre el cultivo de caña de azúcar (2016), se ha consolidado la tabla 1.

\section{Tabla 1. Distribución del índice de Gini y del cultivo de caña de azúcar en Colombia}

\begin{tabular}{c|c|c|c}
\hline Departamento & Gini & Área de caña de azúcar cultivada (ha) \\
\hline Amazonas & 0.991 & 0 \\
\hline Antioquia & 0.8486 & 0 \\
\hline Arauca & 0.8254 & 0 \\
\hline
\end{tabular}

(Continúa) 
Tabla 1. Distribución del índice de Gini y del cultivo de caña de azúcar en Colombia

\begin{tabular}{|c|c|c|}
\hline Departamento & Gini & Área de caña de azúcar cultivada (ha) \\
\hline Atlántico & 0.7385 & 0 \\
\hline Bolívar & 0.7152 & 0 \\
\hline Boyacá & 0.8108 & 0 \\
\hline Caldas & 0.8367 & 3220 \\
\hline Caquetá & 0.5994 & 0 \\
\hline Casanare & 0.8284 & 0 \\
\hline Cauca & 0.8717 & 44479 \\
\hline Cesar & 0.6999 & 0 \\
\hline Chocó & 0.9819 & 0 \\
\hline Córdoba & 0.7862 & 0 \\
\hline Cundinamarca & 0.8028 & 0 \\
\hline Guainía & 0.616 & 0 \\
\hline Guaviare & 0.5519 & 0 \\
\hline Huila & 0.767 & 0 \\
\hline La Guajira & 0.6835 & 0 \\
\hline Magdalena & 0.7118 & 0 \\
\hline Meta & 0.8716 & 17408 \\
\hline Nariño & 0.8681 & 0 \\
\hline Norte de Santander & 0.7123 & 0 \\
\hline Putumayo & 0.7431 & 0 \\
\hline Quindío & 0.8053 & 273 \\
\hline Risaralda & 0.7984 & 2896 \\
\hline $\begin{array}{c}\text { San Andrés, Providencia } \\
\text { y Santa Catalina }\end{array}$ & 0.728 & 0 \\
\hline
\end{tabular}




\section{Tabla 1. Distribución del índice de Gini y del cultivo de caña de azúcar en Colombia}

\begin{tabular}{|c|c|c|}
\hline Departamento & Gini & Área de caña de azúcar cultivada (ha) \\
\hline Santander & 0.7123 & 0 \\
\hline Sucre & 0.7721 & 0 \\
\hline Tolima & 0.7828 & 0 \\
\hline Valle del Cauca & 0.9003 & 187337 \\
\hline Vaupés & 0.4706 & 0 \\
\hline Vichada & 0.6583 & 0 \\
\hline Total & & 255613 \\
\hline
\end{tabular}

Fuente: Elaboración propia con base en datos de UPRA (2016) y MADR (2016).

Se observa que la caña se encuentra en solo 6 departamentos del país, con una participación importante del Valle del Cauca. Reconvirtiendo las variables a dummy para el caso de las de caña de azúcar, podemos analizar si hay alguna diferencia entre los departamentos que tienen esta plantación y los que no, en términos de distribución de la tierra. La tabla 2 presenta lo que la prueba T-test arroja.

\section{Tabla 2. Prueba de hipótesis}

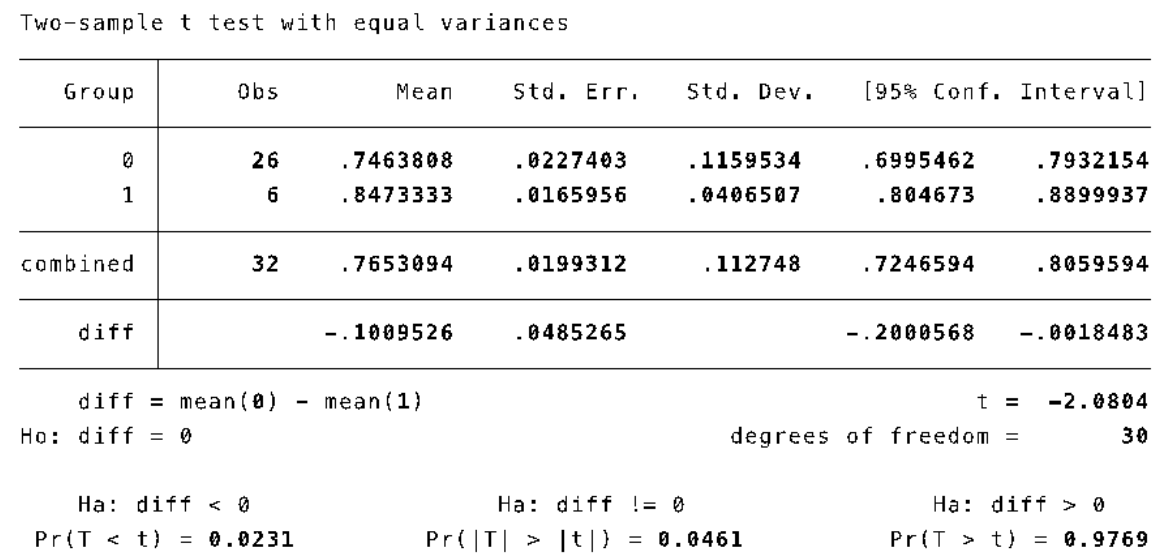

Fuente: Elaboración propia con base en datos de MADR (2016) y UPRA (2016). 
Para este caso, la hipótesis nula asume que no hay diferencia entre las medias de los departamentos que cultivan con aquellos que no lo hacen. Sin embargo, la hipótesis alternativa (si hay diferencia) es estadísticamente significativa $(<0.05)$, por lo que debe rechazarse la hipótesis nula. Esto quiere decir que sí hay una diferencia estadísticamente significativa entre los municipios que siembran caña de azúcar y los que no. En promedio, los departamentos que la siembran tienen un índice de Gini 0.1 más alto que los que no. Haciendo un análisis por municipios, con la misma fuente de información, se encuentra que los 1031 municipios que no cultivan caña en el país tienen un Gini promedio de 0.71, mientras que los 35 que la cultivan tienen un Gini promedio de 0.83.

La tabla 3 ilustra la distribución de los municipios que cultivan caña de azúcar en Colombia.

Tabla 3. Distribución de la caña de azúcar en Colombia

\begin{tabular}{c|c|c}
\hline Municipio & Departamento & Área cultivada (ha) \\
\hline Palmira & Valle & 37134 \\
\hline Candelaria & Valle & 23921 \\
\hline El Cerrito & Valle & 16313 \\
\hline Puerto López & Meta & 17408 \\
\hline Bugalagrande & Valle & 9201 \\
\hline Pradera & Valle & 9225 \\
\hline Jamundí & Valle & 9316 \\
\hline Zarzal & Valle & 8817 \\
\hline Tuluá & Valle & 7846 \\
\hline Florida & Valle & 7809 \\
\hline Puerto Tejada & Cauca & 7810 \\
\hline Guacarí & Valle & 6986 \\
\hline
\end{tabular}


Tabla 3. Distribución de la caña de azúcar en Colombia

\begin{tabular}{|c|c|c|}
\hline Municipio & Departamento & Área cultivada (ha) \\
\hline Guadalajara de Buga & Valle & 6338 \\
\hline Miranda & Cauca & 6238 \\
\hline Obando & Valle & 5646 \\
\hline Santander de Quilichao & Cauca & 6130 \\
\hline Padilla & Cauca & 5542 \\
\hline Cali & Valle & 4652 \\
\hline Corinto & Cauca & 5181 \\
\hline Cartago & Valle & 4429 \\
\hline San Pedro & Valle & 4652 \\
\hline Villa Rica & Cauca & 4757 \\
\hline Yotoco & Valle & $3 \mathrm{i} 705$ \\
\hline Guachené & Cauca & 4163 \\
\hline Riofrío & Valle & 2878 \\
\hline Ginebra & Valle & 3370 \\
\hline Caloto & Cauca & 3829 \\
\hline Viterbo & Caldas & 2857 \\
\hline Andalucía & Valle & 2546 \\
\hline Roldanillo & Valle & 2219 \\
\hline Yumbo & Valle & 2018 \\
\hline Ansermanuevo & Valle & 1843 \\
\hline Balboa & Risaralda & 1633 \\
\hline Toro & Valle & 1935 \\
\hline La Unión & Valle & 1278 \\
\hline Otros & & 6125 \\
\hline
\end{tabular}

Fuente: Elaboración propia con base en datos de UPRA (2016) y MADR (2016). 
Se observa una importante presencia del cultivo en Valle del Cauca y Cauca, departamentos que tienen índices de Gini más altos que la media nacional (0.76, según datos departamentales de UPRA, 2016).

El mapa 1 nos ayuda a comprender mejor la distribución.

\section{Mapa 1. Distribución del cultivo de caña de azúcar}

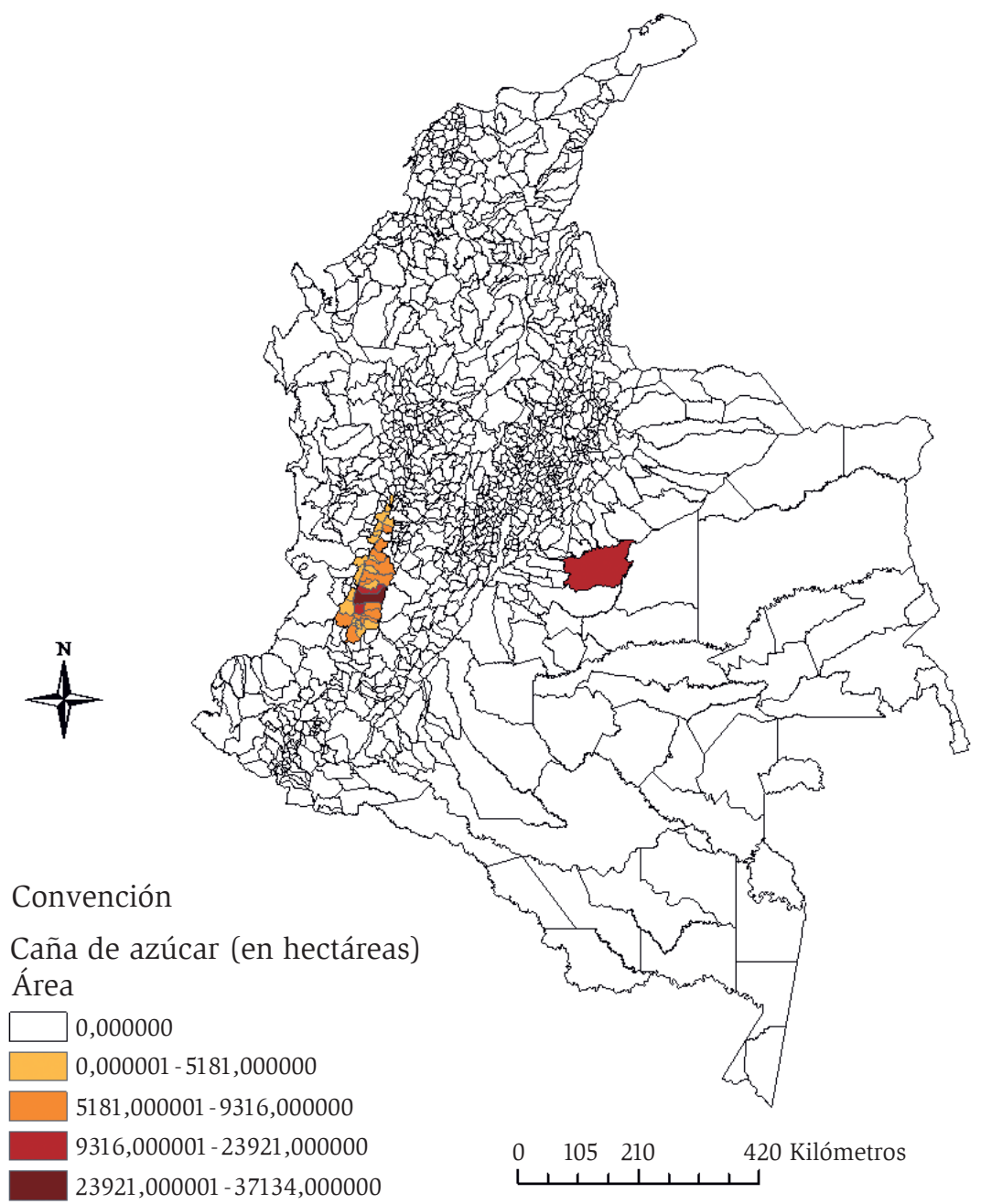

Fuente: Datos del MADR (2016). 
La misma información la podemos plasmar en tres dimensiones:

\section{Mapa 2. Distribución del cultivo de caña de azúcar en tres dimensiones}

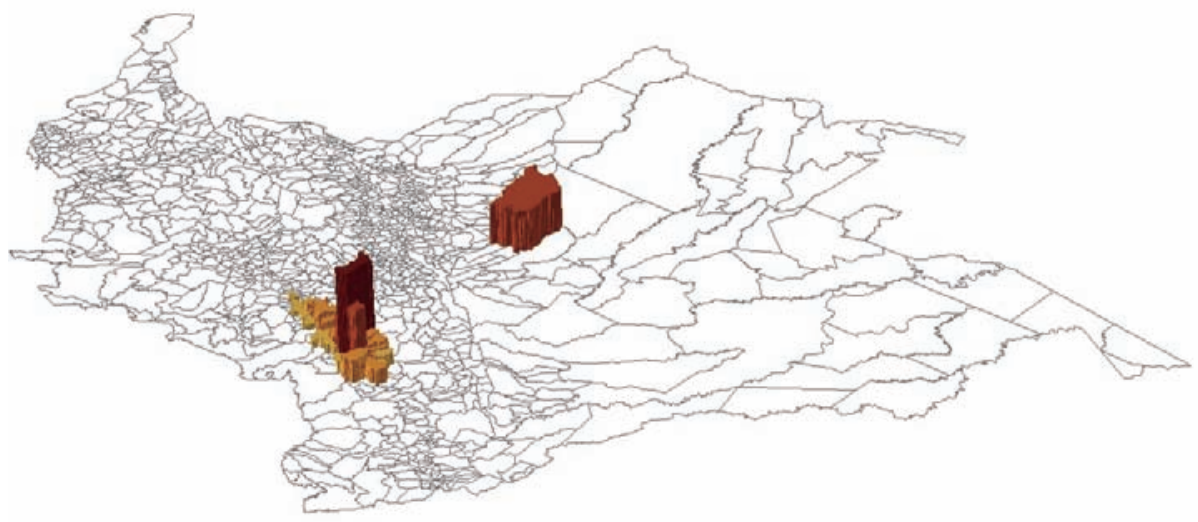

Fuente: Datos del MADR (2016).

Hay evidencia de que la agroindustria de la caña de azúcar ha sido capaz de trasladar el capital económico a términos políticos, de manera que las cabezas del sector tienen amplia influencia sobre las autoridades, incluso las nacionales, debido principalmente a que la producción de etanol las ha revertido de una particular posición estratégica en el desarrollo del país. No resulta extraña, entonces, una política de favorecimiento constante por parte del Estado colombiano (Pérez y Álvarez, 2009). Tal y como se describió previamente, la fuerte promoción que se viene haciendo de los biocombustibles encamina claramente la expansión de la caña, no solo dentro del Valle del Cauca, sino también en la altillanura colombiana (Ayala-Osorio, 2019). El panorama de la caña de azúcar en Colombia es favorable, debido al crecimiento progresivo de la demanda de etanol y a la instalación exitosa de plantas procesadoras en diversas partes de país, incluyendo el Meta (Grande, 2016).

Según Arias (2017), algunos ingenios azucareros también han venido posando sus intereses en la altillanura colombiana, con el fin de ampliar y diversificar su negocio, eso sí, mediante la reproducción de un modelo 
idéntico al del Valle del Cauca, a pesar de la disparidad ambiental y de los correspondientes daños ambientales por ello asociados. De este modo, es válido ahora pasar a abordar la situación de esta subregión.

\section{Una mirada a la altillanura}

La altillanura es una subregión de la Orinoquía que se extiende sobre 13.5 millones de hectáreas, desde el municipio de Puerto López hasta el lado este del río Meta. Está conformada por siete municipios: La Primavera, Cumaribo, Puerto Carreño y Santa Rosalía en Vichada y Puerto López, Puerto Gaitán y Mapiripán en Meta. En esta zona se encuentra la mayor parte del petróleo de la Orinoquía y se concentran la mayoría de las iniciativas agroindustriales (DNP, 2014).

Colombia tiene 21.8 millones de hectáreas potencialmente cultivables. El $5.5 \%$ de estas (1.2 millones de hectáreas) se encuentran en la altillanura. Sin embargo, en la subregión solo se cultivan 80167 hectáreas. Según estimaciones, un total de 2.8 millones de hectáreas pueden utilizarse para la agricultura, la silvicultura y la ganadería. La subregión se caracteriza por la baja productividad por hectárea y los altos costos de producción, que según los documentos oficiales dan como resultado una baja competitividad. Todo esto se debe a las economías de baja escala, los altos precios de los suministros y la falta de tecnología (DNP, 2014).

El gobierno de Colombia ha venido apoyando la implementación del modelo brasileño conocido como el Cerrado en la altillanura, debido en gran parte a las similitudes geográficas, con el objetivo de generar las condiciones necesarias para la expansión de monocultivos, sobre todo en Puerto Gaitán, Puerto López y Cumaribo (Piñeros, 2016).

En los setenta Brasil implementó un modelo agroindustrial en regiones de suelos ácidos y poca fertilidad. Mediante la incorporación de tecnología (principalmente a través de fertilizantes), y de acuerdo con los estándares de producción agrícola, los suelos fueron potencializados para dar paso a la implementación de nuevos cultivos y pasturas (Arraes, Geraldo, Santana y Alves, 2012). En efecto, la sabana brasileña 
cuenta con suelos con bajo ph y elevados contenidos de aluminio. Aún así, por mucho tiempo las poblaciones desarrollaron agricultura de subsistencia, hasta que el gobierno brasileño empezó a promover de manera significativa los proyectos agroindustriales mediante beneficios que incluyen subsidios, incentivos fiscales y préstamos a bajo interés que, sumados todos estos, facilitaron la instalación de un sistema agrícola masivamente mecanizado (Ratter, Ribeiro y Bridgewater, 1997).

Tales proyectos agroindustriales demandan una inyección financiera significativa y propician el acaparamiento de la tierra. Valga decir que para 1985, solo el 4 \% de las fincas tenía más de 1000 hectáreas, pero estas mismas cubrían más del $60 \%$ del área total. Por otro lado, el $50 \%$ de las fincas menores de 50 hectáreas apenas abarcaban el $0.2 \%$ del total de la zona (Ratter et al., 1997). Sin duda esta situación parece adecuarse al patrón de acaparamiento de tierras descrito en el apartado teórico, en el que grandes extensiones de tierra son controladas con el fin de adelantar allí proyectos a gran escala.

Usando datos de las mismas fuentes previamente consultadas, hemos consolidado la tabla 4 sobre la situación en la altillanura:

Tabla 4. Datos sobre la altillanura

\begin{tabular}{c|c|c|c} 
Departamento & Municipio & Gini & Área sembrada (hectáreas) \\
\hline Meta & Mapiripán & 0.7185 & 0 \\
\hline Meta & Puerto Gaitán & 0.7172 & 0 \\
\hline Meta & Puerto López & 0.7751 & 17408 \\
\hline Vichada & Cumaribo & 0.735 & 0 \\
\hline Vichada & La Primavera & 0.508 & 0 \\
\hline Vichada & Puerto Carreño & 0.623 & 0 \\
\hline Vichada & Santa Rosalía & 0.489 & 0 \\
\hline TOTAL & & & 17408
\end{tabular}

Fuente: elaboración propia con base en datos de MADR (2016) y UPRA (2016). 
La media del índice de Gini en la región es de 0.65, quizá debido a la presencia notable de tierras baldías. Sin embargo, Puerto López, que es uno de los principales cultivadores de caña de azúcar en el país, tiene un índice de Gini de 0.77 .

\section{Sobre las tierras baldías}

Los "baldíos" son aquellas tierras que no son propiedad de particulares y, por lo tanto, pertenecen al Estado, según el Código Civil. Dichos bienes están destinados a programas de redistribución llevados a cabo por la Agencia Nacional de Tierras a través de un proceso administrativo agrario por el cual el Estado emite títulos de propiedad a campesinos que tienen bajos ingresos y carecen de otras tierras rurales en el territorio nacional (Ley 160 de 1994; Decreto 902 del 2017). Los baldíos son entonces tierras públicas cuya privatización solo se permite a favor de campesinos pobres a través de un acto administrativo. Privatizarlos por otro medio está completamente prohibido, según la Constitución Política y la Ley 160 de 1994. Sin embargo, se han detectado algunas estrategias sistemáticas para apropiarlas que se estudiarán más adelante.

\section{Los hallazgos de la Contraloría}

Es muy importante destacar la prohibición de ser propietario de predios que tienen sus orígenes en procesos de adjudicación de baldíos si sus extensiones exceden los límites máximos permitidos. En consecuencia, los contratos de compraventa o los actos utilizados para transferir tales tierras se considerarán ilegales si se forma una propiedad que excede la Unidad de Agricultura Familiar (Ley 160 de 1994). Estos requisitos tienen como finalidad establecer objetivos o sujetos de la reforma agraria, excluyendo a aquellos individuos que tienen suficientes recursos para la adquisición privada de tierras o aquellos que tienen propiedades rurales.

En 2014, la Contraloría General de la República (CGR) identificó una acumulación irregular de baldíos en la altillanura realizada por personas naturales y jurídicas que, directamente o por medio de empresas 
creadas con fines específicos, compraron y acumularon dichas tierras. La CGR descubrió que algunos inversionistas crearon diferentes sociedades limitadas por medio de las cuales compraron los antiguos baldíos y solicitaron el Incentivo a la Capitalización Rural.

La investigación encontró que dos tradicionales empresas colombianas de la industria azucarera y sus derivados construyeron varias compañías fachada con el fin de adquirir predios originalmente baldíos. La primera de ellas constituyó 27 compañías con el fin de adquirir 31000 hectáreas de predios originalmente baldíos en los municipios La Primavera y Santa Rosalía en el departamento de Vichada. La segunda compañía adquirió varios predios rurales en San Carlos de Guaroa (Meta), lo mismo que en Orocué (Casanare). En el Meta, la compañía es propietaria de 29 predios rurales que suman 5884 hectáreas excediéndose así la Unidad Agrícola Familiar permitida en la región (entre 623 a 847 hectáreas). La Contraloría concluyó que existió una acumulación de bienes inicialmente adjudicados como baldíos, en contravía de lo dispuesto en la Ley 160 de 1994. En el departamento del Vichada la compañía ha acumulado más de 16829 hectáreas, luego de haber adquirido predios que fueron inicialmente adjudicados como baldíos por el Incora entre 1986 y 2000, algunos de los cuales presentan importantes incrementos en su valor. El área acumulada excede ampliamente la Unidad Agrícola familiar regional (CGR, 2014).

Los hallazgos de la Contraloría indican que hay intereses agroindustriales por la apropiación de tierras baldías en zonas alejadas. En particular, parece haber un interés de parte de la industria de la caña de azúcar que utiliza mecanismos legales para apropiarse de bienes inicialmente públicos, siguiendo así un claro patrón de acumulación por desposesión que, a la larga, deriva en tierras acaparadas. Debe tenerse en cuenta que los baldíos están concebidos para fines de reforma agraria, por lo que su destinación se hace exclusiva para campesinos pobres en los términos del Decreto 902 del 2017. El englobe de estos predios está completamente prohibido, incluso cuando no medie la violencia propia 
de la acumulación primitiva,. En ese caso el concepto de acumulación por desposesión cobra plena validez.

Esta dinámica de acaparamiento de tierras puede incrementar la concentración histórica, medida en términos de Gini. Como se probó estadísticamente, los departamentos que cultivan caña tienen un índice de desigualdad de distribución de la tierra 0.1 más alto en comparación con los que no cultivan. Además, el único municipio que cultiva caña en la altillanura, Puerto López, es el que registra el Gini más alto de la región, circunstancia que puede dar lugar a pensar en algún tipo de asociación entre la caña y el incremento de la desigualdad en la propiedad de la tierra en esa región del país.

\section{Conclusiones}

El caso colombiano exhibe un trasfondo histórico de concentración de la tierra originado desde épocas anteriores a la independencia, pero agravado por una política de adjudicación de baldíos basada en la riqueza y la influencia en desmedro de un enfoque realmente distributivo. Desde los inicios de la República la tierra ya se encontraba concentrada, situación que determinó fuertemente las relaciones en torno a la tierra en Colombia.

El acaparamiento actual de la tierra en el país guarda similitudes con otras regiones del globo. El capitalismo se ha expandido a zonas remotas del llamado sur global, esto a causa de sus condiciones y posibilidades para la producción a gran escala, a expensas de los métodos locales y tradicionales. Los cultivos agroindustriales, como la caña de azúcar, han reemplazado a otros debido a sus múltiples propósitos y usos en la producción de alimentos y combustibles. Este proceso sigue el mismo patrón internacional donde dichos cultivos están fuertemente incentivados por los Estados (Borras et al., 2012). En el caso colombiano, una notable reducción de impuestos y las órdenes para usar ciertos porcentajes 
de biocombustibles en vehículos nuevos deben mencionarse como incentivos obvios que apuntan a expandir este tipo de producción.

El proceso ha seguido una lógica de acaparamiento en el que vastas regiones del Valle del Cauca y la altillanura son controladas con el fin de implementar proyectos a gran escala de caña de azúcar, que como se estudió, sirve como materia prima para varias industrias nacionales y es la única fuente de etanol en Colombia, circunstancia que confiere excepcionales capacidades de negociación y notable influencia a las cabezas de este sector.

El acaparamiento de tierras por parte de la caña de azúcar parece impactar fuertemente la concentración histórica de la tierra en Colombia, particularmente en el Valle del Cauca, en donde las tierras de comunidades negras y campesinas han sido históricamente apropiadas por las élites blancas. Así lo sugieren las dinámicas de apropiación encontradas en el departamento, donde un alto índice de Gini, medido en términos de propiedad de la tierra, coincide con una notable presencia de caña de azúcar. En una línea similar, Puerto López, el tercer mayor cultivador de caña de azúcar en Colombia, tiene el índice Gini más alto en altillanura.

Según Harvey (2003), el concepto de acumulación primitiva no explica completamente la acumulación contemporánea, ya que podrían estar presentes otras dinámicas distintas de la violencia. Por lo tanto, el concepto de acumulación por desposesión debe ser incorporado. En el caso colombiano, la CGR ha demostrado cómo los grandes inversionistas utilizaron mecanismos aparentemente legales para acaparar tierras y desarrollar proyectos a gran escala, incluso mediante la captura de otros recursos del Estado.

La CGR descubrió que los actores agroindustriales han venido apropiándose de predios inicialmente adjudicados como baldíos, lo cual está totalmente prohibido, utilizando mecanismos legales complejos, 
como la creación de diferentes personas jurídicas ficticias para la compra de los predios. Estas estrategias implican la privatización de los bienes sociales según los elementos del concepto de acumulación por desposesión, en la medida en que los reales beneficiarios, en este caso campesinos pobres, son privados de su derecho a la tierra mediante compras con fines de acaparamiento completamente proscritas. Los actores detrás de tales estrategias tienen conexiones a largo plazo con los biocombustibles, particularmente con la caña de azúcar, y algunos de ellos son ampliamente conocidos en Colombia.

\section{Referencias}

Arias, Wilson (2017). Así se roban la tierra en Colombia. Bogotá: Impresol.

Arraes Pereira, Pedro; Geraldo Martha; Santana, Carlos y Alves, Eliseu. (2012). The development of Brazilian agriculture, future technological challenges and opportunities. Agriculture \& Food Security, 1(4), 2-12. DOI: 10.1186/2048-7010-1-4.

Ayala-Osorio, Germán (2019). El monocultivo de la caña de azúcar en el valle geográfico del río Cauca (Valle del Cauca, Colombia): un enclave que desnaturaliza la vida ecosistémica. FORUM. Revista Departamento Ciencia Política, 15, 37-66. DOI: https://doi.org/10.15446/frdcp.n15.72452.

Borras, Saturnino; Franco, Jennifer; Gómez, Sergio; Kay, Cristobal y Spoor, Max (2012). Land grabbing in Latin America and the Caribbean. The Journal of Peasant Studies, 39(3-4), 845-872, DOI: 10.1080/03066150.2012.679931.

Borras, Saturnino; Kay, Cristóbal; Gómez, Sergio y Wilkinson, John (2013). Acaparamiento de tierras y acumulación capitalista: aspectos clave en América Latina. Revista Interdisciplinaria de Estudios Agrarios, 38(1), 75-103.

Borras, Saturnino; Hall, Ruth; Edelman, Marc; Scoones, Ian; White, Ben y Wolford, Wendy (2015). Resistance, acquiescence or incorporation? An introduction to land grabbing and political reactions 'from below'. The Journal of Peasant Studies, 42:3-4, 467-488, DOI: 10.1080/03066150.2015.1036746.

Comisión Económica de las Naciones Unidas para América y el Caribe [CEPAL] (2002). El conglomerado del azúcar del Valle del Cauca, Colombia. Serie Desarrollo productivo. Santiago de Chile: UN. 
Programa de las Naciones Unidas para el Desarrollo [PNUD] (2011). Informe Nacional de Desarrollo Humano. Bogotá: INDH PNUD.

Contraloría General de la República [CGR] (2014). Acumulación irregular de predios baldios en la altillanura colombiana. Informe de actuación especial. Bogotá: CGR.

De Angelis, Massimo (2000). Marx's Theory of Primitive Accumulation: A Suggested Reinterpretation. Londres: University of East London.

Delgado, Juan; Salgado, José y Perez, Ronaldo (2015). Perspectivas de los biocombustibles en Colombia. Revista Ingenierías, 14(27), 13-28. http:// dx.doi.org/10.16924\%2Friua.v0i29.252

Departamento Administrativo Nacional de Estadística [DANE] (2015). Cuenta satélite piloto de la agroindustria del cultivo de caña de azúcar 2005-2012. Bogotá, Colombia.

Departamento Nacional de Planeación [DNP] (2014). Consejo Nacional de Política Económica y Social. República de Colombia. Documento CONPES 3797. Bogotá, Colombia.

Edelman, Marc y León, Andrés (2013). Cycles of Land Grabbing in Central America: an argument for history and a case study in the Bajo Aguán, Honduras. Third World Quarterly, 34(9), 1697-1722, DOI: 10.1080/01436597.2013.843848.

Escuela Itinerante Afronortecaucana y Grupo Semillas (2013). Transformaciones de las fincas norte-caucanas para la persistencia en el territorio. Cuaderno de Semillas, 3, 2-10.

Fajardo, Darío (diciembre, 2001) La tierra y el poder político; la reforma agraria y la reforma rural en Colombia. Ponencia presentada en el Seminario permanente sobre problemas agrarios y rurales: Proyecto "Viabilidad y reconstrucción de la sociedad rural colombiana”. Bogotá.

(julio 17-19, 2002). Situación y perspectivas del desarrollo rural en el contexto del conflicto colombiano. Documento presentado ante el seminario Situación y perspectivas para el desarrollo agrícola y rural en Colombia. Santiago de Chile.

Franco, Jennifer (2012). La carrera mundial para hacer más "transparente" el acaparamiento de tierras. En Acaparamiento de tierras, el nuevo expolio. Editado por el Centro de Investigación para la Paz. 18-26. Madrid: FUHEM. 
Glassman, Jim (2006). Primitive accumulation, accumulation by dispossession, accumulation by 'extra-economic' means. Progress in Human Geography, 30(5), 608-625.

Gilbert, Jérémie (2017). Land grabbing, investments \& indigenous peoples' rights to land and natural resources: case studies and legal analysis. Copenhangen: International work group for indigenous affairs.

Gómez, Sergio (Ed.) (2014). The land market in Latin America and the Caribbean. Santiago de Chile, Chile: Food and Agriculture Organization [FAO].

Grande, Carlos (2016). Residuos agroindustriales biocombustibles. Cali: Universidad de San Buenaventura - Lemoine Editores.

Hallam, David (2009). International Investments in agricultural production. En Kugelman, Michael y Levenstein, Susan (Eds.), LAND GRAB? The Race for the World's Farmland. Wodrow Wilson International Center for Scholars. Washington, U.S.: Wodrow Wilson International Center for Scholars.

Harvey, David (2003). The New Imperialism. Great Britain: Oxford University press.

(2006). The limits to capital. U.K.: Verso.

(2010). A Companion to Marx's Capital. U.K. and U.S.: Verso.

Hurtado Teodora (2001). La protesta social en el Norte del Cauca y el surgimiento de la movilización étnica afrocolombiana. En Pardo, Mauricio (edit.), Acción colectiva, Estado y etnicidad en el Pacífico colombiano. Bogotá: Colciencias, Instituto Colombiano de Antropología e Historia.

Ibáñez, Ana María y Velásquez, Andrea (2008). El impacto del desplazamiento forzoso en Colombia: condiciones socioeconómicas de la población desplazada, vinculación a los mercados laborales y políticas públicas. Santiago de Chile, Chile: CEPAL.

Ibáñez, Ana maría; Muñoz, Juan; Gáfaro, María; Arias, María; Gómez, Iván; Granados, Jorge; Cuellar, Diana et al. (2012). Atlas de la distribución de la propiedad rural en Colombia. Bogotá: Instituto Geográfico Agustín Codazzi [IGAC], Universidad de los Andes, Universidad de Antioquia y Gobernación de Antioquia. 
Instituto Geográfico Agustín Codazzi [IGAC] (4 de febrero de 2011). Resolución 070 de 2011.

Levien, Michael (2011a). Special economic zones and accumulation by dispossession in India. Journal of Agrarian Change, 11(4), 454-483.

(2011b, April 6-8). The land question: special economic zones and the political economy of disposession in India. Ponencia presentada en la Conferencia internacional sobre acaparamiento global de tierras. La Haya: Land Deals Politics Initiative.

(2012). The land question: special economic zones and the political economy of dispossession in India. Journal of Peasant Studies, 39(3-4), 933-969.

(2013). The Politics of Dispossession: Theorizing India's “Land Wars”. Politics \& Society. 41(3), 351-394.

Machado, Absalón (2009). Ensayos para la historia de la política de tierras en Colombia. Bogotá, Colombia: Universidad Nacional de Colombia.

Matondi, Prosper; Havnevik, Kjell y Beyene, Atakilte (2011). Biofuels, land grabbing and food security in Africa. Upsala: The Nordic Africa Institute.

Ministerio de Agricultura y Desarrollo Rural [MADR] (2016). Área sembrada y área cosechada del cultivo de caña azucarera 2007-2016. Recuperado de: https://bit.ly/2pjn2VV

Marx, Karl (1867). Capital. A Critique of Political Economy. London, England.

Pérez, Mario y Álvarez, Paula (2009). Deuda social y ambiental del negocio de la caña de azúcar en Colombia. Bogotá: ARFO editores.

Perfetti, Juan y Saavedra, Víctor (junio, 2017). Acuerdo de Paz y Decreto Ley de Tierras. Economía y Política. Fedesarrollo. 69. Recuperado de: https:// bit.ly/2NsbvRH

Piñeros, Robinzon (2016). La territorialización del agronegocio de la Palma de Aceite y la Caña de Azúcar en la altillanura colombiana. Aportes para el estudio de sus efectos a las condiciones de trabajo de los asalariados rurales. DOI: 10.13140/RG.2.1.2214.2321. Recuperado de: https://bit.ly/2z5XRux 
Ratter James; Ribeiro, José y Bridgewater, Samuel (1997). The Brazilian Cerrado Vegetation and threats to its Biodiversity. Annals of botany, 80, 223-230.

Rodríguez, Diana y Cepeda Edilberto (junio de 2011). Concentración de la tierra en Colombia. Comunicaciones en Estadística, 4(1), 29-42.

Salinas, Yamile (2012). El caso de Colombia. En Soto, Fernando y Gómez, Sergio. Dinámicas del mercado de la tierra en América Latina y el Caribe: Food and Agriculture Organization [FAO].

Seo, Kihuan y Rodríguez, Natalia (2012). Land Grab, Food Security and Climate Change: A Vicious Circle in the Global South. En Chhetri, Netra (ed). Human and Social Dimensions of Climate Change. Intechopen, DOI: $10.5772 / 50876$.

Ulas, Onur (2017). Between equal rights: Primitive accumulation and capital's violence. Political Theory. Research Collection School of Social Sciences, 46(6): 885-914.

Unidad de Planeación Rural Agropecuaria [UPRA]. (2016). Análisis de la distribución de la propiedad rural en Colombia. Propuesta metodológica. Bogotá, Colombia: UPRA.

Uribe-Castro, Hernando (2014). Expansión cañera en el Valle del Cauca y resistencias comunitarias (Colombia). Ambiente y Sostenibilidad, 4, 16-30.

Vega, Alejandra; Moncaleano, Jorge; Ortiz, Stefan; Carvajalino, Martha y Rodríguez, Flavio (2017). Concentración y extranjerización de tierras productivas en Colombia. Bogotá, Colombia: Food and Agriculture Organization [FAO].

Vogelgesang, Frank. (2003). Derechos de propiedad, costos de transacción, externalidades y mercados de tierras rurales en América Latina y el Caribe. En Tejo, Pedro (comp.), Mercados de tierras agrícolas en América Latina y el Caribe: una realidad incompleta. Santiago de Chile, Chile: Comisión Económica de las Naciones Unidas para América y el Caribe [CEPAL].

White, Ben (2012). Transacciones de tierras, desposesión y el futuro de la agricultura. En Centro de Investigación para la Paz (Ed.) Acaparamiento de tierras, el nuevo expolio (pp.18-26). Madrid: FUHEM. 